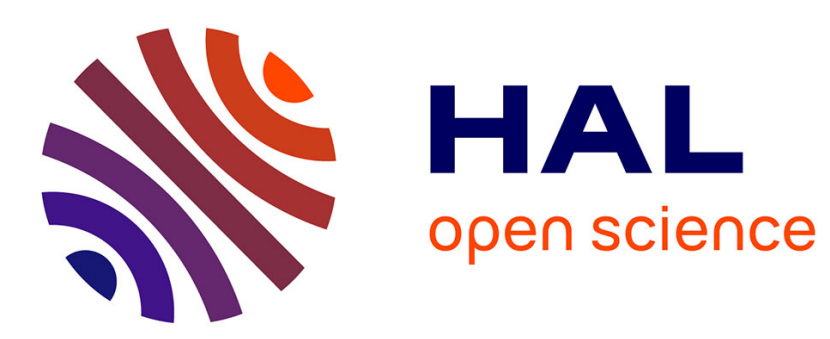

\title{
Slowing Allee effect versus accelerating heavy tails in monostable reaction diffusion equations
}

Matthieu Alfaro

\section{To cite this version:}

Matthieu Alfaro. Slowing Allee effect versus accelerating heavy tails in monostable reaction diffusion equations. Nonlinearity, 2017, 30 (2), pp.687-702. 10.1088/1361-6544/aa53b9 . hal-01149354

\section{HAL Id: hal-01149354 \\ https://hal.science/hal-01149354}

Submitted on 6 May 2015

HAL is a multi-disciplinary open access archive for the deposit and dissemination of scientific research documents, whether they are published or not. The documents may come from teaching and research institutions in France or abroad, or from public or private research centers.
L'archive ouverte pluridisciplinaire HAL, est destinée au dépôt et à la diffusion de documents scientifiques de niveau recherche, publiés ou non, émanant des établissements d'enseignement et de recherche français ou étrangers, des laboratoires publics ou privés. 


\title{
Slowing Allee effect vs. accelerating heavy tails in monostable reaction diffusion equations
}

\author{
Matthieu Alfaro ${ }^{1}$.
}

\begin{abstract}
We focus on the spreading properties of solutions of monostable reaction-diffusion equations. Initial data are assumed to have heavy tails, which tends to accelerate the invasion phenomenon. On the other hand, the nonlinearity involves a weak Allee effect, which tends to slow down the process. We study the balance between the two effects. For algebraic tails, we prove the exact separation between "no acceleration and acceleration". This implies in particular that, for tails exponentially unbounded but lighter than algebraic, acceleration never occurs in presence of an Allee effect. This is in sharp contrast with the KPP situation [19]. When algebraic tails lead to acceleration despite the Allee effect, we also give an accurate estimate of the position of the level sets.
\end{abstract}

Key Words: reaction diffusion equations, spreading properties, heavy tails, Allee effect, acceleration.

AMS Subject Classifications: 35K57, 35B40, 92D25.

\section{Introduction}

In this paper we are concerned with the spreading properties of $u(t, x)$ the solution of the monostable reaction-diffusion equation

$$
\partial_{t} u=\partial_{x x} u+f(u), \quad t>0, x \in \mathbb{R},
$$

when the initial data is front-like and has a heavy tail. When the nonlinearity $f$ is of the Fisher-KPP type, Hamel and Roques [19] proved that such solutions spread by accelerating and precisely estimated the position of the level sets of $u(t, \cdot)$ as $t \rightarrow \infty$, revealing that they are propagating exponentially fast. The goal of the present paper is to introduce a weak Allee effect, by letting $f^{\prime}(0)=0$, and study the balance between such a slowing effect and the acceleration that heavy tails tend to induce. We prove that, for data with heavy tails but lighter than algebraic, acceleration is cancelled by any weak Allee effect (even if very small). For algebraic tails, we prove the exact separation between "no acceleration and acceleration". In the latter case, we estimate the position of the level sets of $u(t, \cdot)$ as $t \rightarrow \infty$, revealing that they are propagating polynomially fast.

\footnotetext{
${ }^{1}$ I3M, Université de Montpellier 2, CC051, Place Eugène Bataillon, 34095 Montpellier Cedex 5, France. E-mail: matthieu.alfaro@univ-montp2.fr
} 
Heavy tails in the Fisher-KPP context. In some population dynamics models, a common assumption is that the growth is only slowed down by the intra-specific competition, so that the growth per capita is maximal at small densities. This leads to consider the reaction diffusion equation (1) — where the quantity $u$ stands for a normalized population density with nonlinearities $f$ of the Fisher-KPP type, namely

$$
f(0)=f(1)=0, \quad \text { and } \quad 0<f(s) \leq f^{\prime}(0) s, \quad \forall s \in(0,1) .
$$

The simplest example $f(s)=s(1-s)$ was first introduced by Fisher [13] and Kolmogorov, Petrovsky and Piskunov [25] to model the spreading of advantageous genetic features in a population.

In such situations, it is well known that the way the front like initial data - in the sense of Assumption 2.1- approaches zero at $+\infty$ is of dramatic importance on the propagation, that is the invasion for large times of the unstable steady state $u \equiv 0$ by the stable steady state $u \equiv 1$. To describe such phenomenon, one can use the notion of spreading speed (if it exists): for a given front like initial data, we say that $c=c\left(u_{0}\right) \in \mathbb{R}$ is the spreading speed of the solution $u(t, x)$ of $(1)$ if

$$
\min _{x \leq v t} u(t, x) \rightarrow 1 \text { as } t \rightarrow \infty \text { if } v<c, \quad \max _{x \geq v t} u(t, x) \rightarrow 0 \text { as } t \rightarrow \infty \text { if } v>c .
$$

For initial data with a exponentially bounded tail (or light tail) at $+\infty$, there is a spreading speed $c \geq c^{*}:=2 \sqrt{f^{\prime}(0)}$ which is selected by the rate of decay of the tail. More precisely, if $u_{0}(x)=\mathcal{O}\left(e^{-\sqrt{f^{\prime}(0)} x}\right)$ as $x \rightarrow+\infty$ (including left compactly supported initial data) then $c=c^{*}=2 \sqrt{f^{\prime}(0)}$, whereas if $u_{0}(x)$ decays like $e^{-\lambda x}, 0<\lambda<\sqrt{f^{\prime}(0)}$, then $c=\lambda+\frac{f^{\prime}(0)}{\lambda}>c^{*}$. There is a large literature on such results and improvements. Let us mention among others the works [13], [25], [27], [17], [21], [31], [3], [24], [7, 8], [6], [20] and the references therein. More recently, the authors in [18] considered the case when the initial data is trapped between two exponentially decreasing tails, revealing further properties which enforce to reconsider the notion of spreading speed.

On the other hand, Hamel and Roques [19] recently considered the case of initial data with heavy tail (or not exponentially bounded), namely

$$
\lim _{x \rightarrow+\infty} u_{0}(x) e^{\varepsilon x}=0, \quad \forall \varepsilon>0 .
$$

Typical examples are algebraic tails but also "lighter heavy tails", see (13) or (14), and "very heavy tails", see (19). In this context, it is then proved in [19] that, for any $\lambda \in(0,1)$, the $\lambda$ level set of $u(t, \cdot)$ travel infinitely fast as $t \rightarrow \infty$, thus revealing an acceleration phenomenon (which in particular prevents the existence of a spreading speed). Also, the location of these level sets is estimated in terms of the heavy tail of the initial data.

Related results exist for the integro-differential equation of the KPP type

$$
\partial_{t} u=J * u-u+f(u),
$$

where the kernel $J$ allows to take into account rare long-distance dispersal events. Here, the initial data is typically compactly supported and this is the tail of the dispersion kernel $J$ that determines how fast is the invasion. If the kernel is exponentially bounded, then propagation occurs at a constant speed, as can be seen in [32], [11], [12] among others. 
More recently, Garnier [14] proved an acceleration phenomenon for kernels which are not exponentially bounded, so that (2) is an accurate model to explain the Reid's paradox of rapid plant migration (see [14] for references on this issue).

To conclude on acceleration phenomena in Fisher-KPP type equations, let us mention the case when the Laplacian is replaced by the generator of a Feller semigroup, a typical example being

$$
\partial_{t} u=-\left(-\partial_{x x}\right)^{\alpha} u+f(u), \quad 0<\alpha<1,
$$

where $-\left(-\partial_{x x}\right)^{\alpha}$ stands for the Fractional Laplacian, whose symbol is $|\xi|^{2 \alpha}$. In this context, it was proved by Cabré and Roquejoffre [9] that, for a compactly supported initial data, acceleration always occurs, due to the algebraic tails of the Fractional Laplacian.

Heavy tails vs. Allee effect. In population dynamics, due for instance to the difficulty to find mates or to the lack of genetic diversity at low density, the KPP assumption is unrealistic in some situations. In other words, the growth per capita is no longer maximal at small densities, which is referred to as an Allee effect.

Remark 1.1. In the sequel, by Allee effect, we always mean weak Allee effect. To take into account a strong Allee effect, for which the growth of the population is negative at small densities, the common nonlinearity is of the bistable type. In such a framework, heavy tails typically do not lead to acceleration [4], [10], [16], [1].

In this Allee effect context, if $f^{\prime}(0)>0$ the situation - even if more complicated - is more or less comparable to the KPP situation: most of the above qualitative results remain valid. On the other hand, much less is known in the degenerate situation where $f^{\prime}(0)=0$, for which typical nonlinearities take the form

$$
f(s)=r s^{\beta}\left(1-s^{\delta}\right), \quad r>0, \beta>1, \delta>0 .
$$

In this work, we focus on the local equation (1) with such an Allee effect. The first question which arises is whether or not propagation (in the sense of invasion) still occurs for equation (1). It turns out that, in some situations, quenching may occur. This happens typically when a compactly supported initial data is too small (in some $L^{1}$ sense) and $\beta>3$. On the other hand, any compactly supported initial data not too small (in some $L^{1}$ sense) will lead to invasion in the sense that

$$
\lim _{t \rightarrow \infty} u(t, x)=1, \quad \text { locally uniformly in space. }
$$

Such results were proved by [33], [5], [37]. See also earlier works [22], [29], [34] for the case when the nonlinearity is of the ignition type.

Since we will consider front like initial data (see below for a precise statement), invasion will always occur. A natural question is therefore to study the balance between the Allee effect (whose strength is measured by $\beta>1$ ) which tends to slow down the invasion process, and heavy tails which tend to accelerate. Let us mention some numerical results [30], [23] for the nonlinearity $f(s)=s^{2}(1-s)$. Also, for nonlinearities $f(s)=s^{\beta}(1-s)$ and algebraic initial data, matched asymptotic expansions [28], [26] have been used to determine if the solution travels with finite or infinite speed.

In this work, we provide a rigorous description of the competition between the Allee effect and the heavy tail for equation (1). For algebraic tails, we prove the separation between 
acceleration or not (depending on the strength of the Allee effect). Also, when acceleration occurs, we precisely estimate the location of the level sets of the solution. This separation for algebraic tails immediately implies that acceleration never occurs for lighter tails (even if the Allee effect is very small), and always occurs for heavier tails (even if the Allee effect is very large). This is in sharp contrast with the KPP situation [19].

As far as the integro-differential equation (2) with an Allee effect is concerned, the question of propagation or not has been recently studied by [35], [36]. One may then wonder what is the exact balance between the Allee effect and dispersion kernels with heavy tails. We refer to [2] for first results in this direction.

Last, notice that the question of acceleration or not in the nonlocal equation (3) with an Allee effect has been recently solved by Gui and Huan [15]. Considering $\partial_{t} u=-\left(-\partial_{x x}\right)^{\alpha} u+$ $u^{\beta}(1-u)$, they show that: for $0<\alpha \leq 1 / 2$ acceleration always occurs whatever $\beta>1$ by comparing with an ignition type problem; next, for $1 / 2<\alpha<1$, acceleration occurs if and only if $\beta<\frac{2 \alpha}{2 \alpha-1}$. See [15] for more precise results.

\section{Assumptions and main results}

Through this work, and even if not recalled, we always assume the following on the initial condition. Notice that, in each result, we clearly state the heavy tail assumption which is therefore not included below.

Assumption 2.1. (Initial condition) The initial condition $u_{0}: \mathbb{R} \rightarrow[0,1]$ is uniformly continuous and asymptotically front-like, in the sense that

$$
u_{0}>0 \text { in } \mathbb{R}, \quad \liminf _{x \rightarrow-\infty} u_{0}(x)>0, \quad \lim _{x \rightarrow+\infty} u_{0}(x)=0 .
$$

Even if not recalled, we always assume the following on the nonlinearity $f$. Notice that, in each result, we clearly quantify the degeneracy assumption (Allee effect) which is therefore not included below.

Assumption 2.2. (Degenerate monostable nonlinearity) The nonlinearity $f:[0,1] \rightarrow \mathbb{R}$ is of the class $C^{1}$, and is of the monostable type, in the sense that

$$
f(0)=f(1)=0, \quad f>0 \text { in }(0,1) .
$$

The steady state 0 is degenerate, that is $f^{\prime}(0)=0$.

The simplest example of such a degenerate monostable nonlinearity is given by $f(s)=$ $s^{\beta}(1-s)$, with $\beta>1$ (in contrast with the KPP situation $\beta=1$ ).

In the sequel, we always denote by $u(t, x)$ the solution of (1) with initial condition $u_{0}$. From the above assumptions and the comparison principle, we immediately get

$$
0<u(t, x)<1 \quad \forall(t, x) \in(0, \infty) \times \mathbb{R} .
$$

Also, as announced in the introduction, the state $u \equiv 1$ does invade the whole line $\mathbb{R}$ as $t \rightarrow \infty$. Indeed, define $\eta:=\inf _{x \leq 0} u_{0}(x)>0$. In view of [37, Theorem 1.1], the solution $v(t, x)$ of (1) with initial data $v_{0}(x)=\eta \chi_{(-\infty, 0)}(x)$ satisfies $\lim _{t \rightarrow \infty} \inf _{x \leq \gamma t} v(t, x)=1$ for some $\gamma>0$. From $v_{0} \leq u_{0}$ and the comparison principle, the same holds true for $u(t, x)$ :

$$
\lim _{t \rightarrow \infty} \inf _{x \leq \gamma t} u(t, x)=1,
$$


so that propagation is at least linear. Notice also that the proof of [19, Theorem 1.1 part a)] does not require the KPP assumption and can then be reproduced to get

$$
\lim _{x \rightarrow+\infty} u(t, x)=0, \quad \forall t \geq 0 .
$$

In order to state our results we define, for any $\lambda \in(0,1)$ and $t \geq 0$,

$$
E_{\lambda}(t):=\{x \in \mathbb{R}: u(t, x)=\lambda\}
$$

the $\lambda$ level set of $u(t, \cdot)$. In view of (5) and (6), for any $\lambda \in(0,1)$, there is a time $t_{\lambda}>0$ such that

$$
\emptyset \neq E_{\lambda}(t) \subset(\gamma t,+\infty), \quad \forall t \geq t_{\lambda} .
$$

Our first main result states that, for algebraic initial tail, acceleration can be blocked by a strong enough Allee effect.

Theorem 2.3. (Cancelling acceleration by Allee effect) Let $\alpha>0$ and $\beta>1$ be such that

$$
\beta \geq 1+\frac{1}{\alpha} .
$$

Assume that there are $C>0$ and $x_{0}>1$ such that

$$
u_{0}(x) \leq \frac{C}{x^{\alpha}}, \quad \forall x \geq x_{0} .
$$

Assume that there are $r>0, \delta>0$ and $s_{0} \in(0,1)$ such that

$$
f(s) \leq r s^{\beta}\left(1-s^{\delta}\right), \quad \forall 0 \leq s \leq s_{0} .
$$

Then, there is a speed $c>0$ such that, for any $\lambda \in(0,1)$, there is a time $T_{\lambda} \geq t_{\lambda}$ such that

$$
\emptyset \neq E_{\lambda}(t) \subset(\gamma t, c t), \quad \forall t \geq T_{\lambda} .
$$

On the one hand, for any Allee effect $\beta>1$, one can find some initial conditions with algebraic tail (whose power is large enough) so that the solutions do not accelerate, as can be seen from (11). On the other hand, for any initial condition with algebraic tail, one can find some Allee effect (strong enough) so that acceleration is cancelled. This is in sharp contrast with the KPP situation $\beta=1$ studied in [19].

Another difference with [19] is concerned with heavy tails that are lighter than algebraic ones, for which acceleration is always cancelled whatever the strength of the Allee effect.

Corollary 2.4. (Heavy tails lighter than algebraic) Let $\beta>1$ be arbitrary. Assume that for all $\alpha>0$, there are $C_{\alpha}>0$ and $x_{0}^{\alpha}>1$ such that

$$
u_{0}(x) \leq \frac{C_{\alpha}}{x^{\alpha}}, \quad \forall x \geq x_{0}^{\alpha} .
$$

Assume (10). Then, for any $\lambda \in(0,1)$, the no acceleration result (11) holds. 
The above result is independent on $\beta>1$ and is valid, among others, for initial data satisfying

$$
u_{0}(x) \leq C e^{-a x /(\ln x)}, \quad \forall x \geq 2, \quad \text { for some } C>0, a>0,
$$

or

$$
u_{0}(x) \leq C e^{-a x^{b}}, \quad \forall x \geq 1, \quad \text { for some } C>0, a>0,0<b<1 .
$$

For such tails, any Allee effect cancels the acceleration, whereas in the KPP case acceleration always occurs [19]. The proof of Corollary 2.4 is obvious: for a given $\beta>1$, select a large $\alpha>0$ such that (8) holds, and then combine (12) with Theorem 2.3 .

From Theorem 2.3 and Corollary 2.4, "the transition from no acceleration to acceleration" seems to take place for algebraic tails. This is confirmed by our next main result, which is concerned with the case when the Allee effect is not strong enough to prevent the acceleration induced by algebraic tails.

Theorem 2.5. (Acceleration despite Allee effect) Let $\alpha>0$ and $\beta>1$ be such that

$$
\beta<1+\frac{1}{\alpha} .
$$

Assume that there are $C>0$ and $x_{0}>1$ such that

$$
u_{0}(x) \geq \frac{C}{x^{\alpha}}, \quad \forall x \geq x_{0} .
$$

Assume that there are $r>0, \delta>0$ and $s_{0} \in(0,1)$ such that

$$
f(s) \geq r s^{\beta}\left(1-s^{\delta}\right), \quad \forall 0 \leq s \leq s_{0} .
$$

Then, for any $\lambda \in(0,1)$, any small $\varepsilon>0$, there is a time $T_{\lambda, \varepsilon} \geq t_{\lambda}$ such that

$$
E_{\lambda}(t) \subset\left(x^{-}(t),+\infty\right) \quad \forall t \geq T_{\lambda, \varepsilon}, \quad x^{-}(t):=\left((r-\varepsilon) C^{\beta-1}(\beta-1) t\right)^{\frac{1}{\alpha(\beta-1)}} .
$$

Combining Theorem 2.3 and Theorem 2.5, we get a complete picture of the propagation phenomenon. Indeed, in the $(\alpha, \beta)$ plane there is no acceleration above or on the hyperbola $\beta=1+\frac{1}{\alpha}$. On the other hand, strictly below the hyperbola acceleration occurs (see Figure $1)$.

As an immediate corollary of Theorem 2.5 we get that, for tails heavier than algebraic ones, acceleration always occurs whatever the strength $\beta>1$ of the Allee effect. Typical examples of such tails are

$$
u_{0}(x) \geq \frac{C}{(\ln x)^{b}}, \quad \forall x \geq 2, \quad \text { for some } C>0, b>0 .
$$

Our last result consists in providing upper bounds on the level sets of $u(t, x)$, when the algebraic tail is stronger than the Allee effect so that acceleration occurs. Combining with the lower bounds of Theorem 2.5, this yields an accurate "sandwich" of the level sets.

Theorem 2.6. (Sandwich of the accelerating level sets) Let $\alpha>0, \delta>0$ and $\beta>1$ be such that (15) holds. Assume that there are $C>0, \bar{C}>0$ and $x_{0}>1$ such that

$$
\frac{\bar{C}}{x^{\alpha}} \geq u_{0}(x) \geq \frac{C}{x^{\alpha}}, \quad \forall x \geq x_{0}
$$




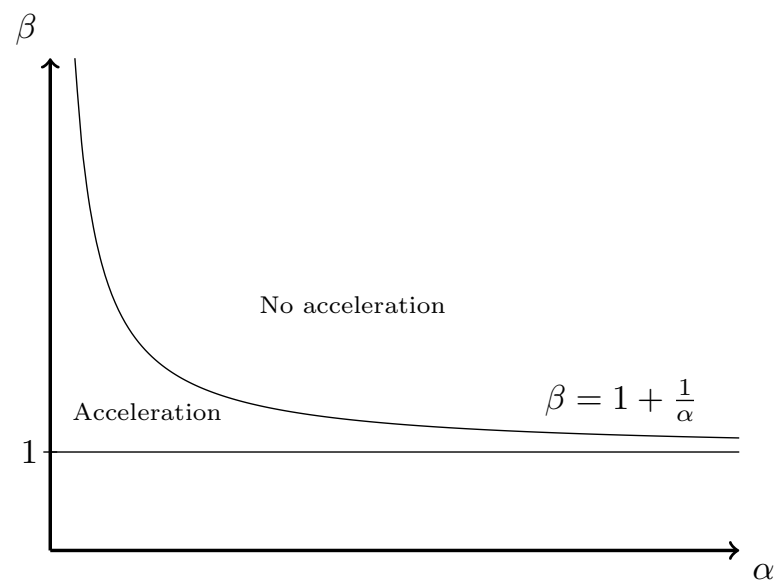

Figure 1: $\beta$-Allee effect vs. $\alpha$-algebraic tail.

Assume (17) and that there is $\bar{r}>0$ such that

$$
\bar{r} s^{\beta} \geq f(s), \quad \forall 0 \leq s \leq 1 .
$$

Then, for any $\lambda \in(0,1)$, any small $\varepsilon>0$, there is a time $\bar{T}_{\lambda, \varepsilon} \geq t_{\lambda}$ such that

$$
E_{\lambda}(t) \subset\left(x^{-}(t), x^{+}(t)\right), \quad \forall t \geq \bar{T}_{\lambda, \varepsilon},
$$

where

$$
x^{-}(t):=\left((r-\varepsilon) C^{\beta-1}(\beta-1) t\right)^{\frac{1}{\alpha(\beta-1)}}, \quad x^{+}(t):=\left((\bar{r}+\varepsilon) \bar{C}^{\beta-1}(\beta-1) t\right)^{\frac{1}{\alpha(\beta-1)}} .
$$

The organization of the paper is as follows. In Section 3 we consider the situation where the Allee effect is stronger than the algebraic tail so that acceleration does not occur, that is we prove Theorem 2.3. In Section 4 we consider the opposite situation, proving the acceleration as stated in Theorem 2.5. Last, in Section 5, we prove the upper estimates on the spreading of the level sets when accelerating, thus completing the proof of Theorem 2.6.

\section{Cancelling acceleration by Allee effect}

In this short section, we prove Theorem 2.3. The formal argument is very simple, close to the rigorous proof and enlightening. We therefore take the liberty to present it: in order to block acceleration, we aim at finding a speed $c>0$ and a power $p>0$ such that $w(z):=\frac{1}{z^{p}}$ is a supersolution of the associated traveling wave equation for $z>>1$, that is

$$
w^{\prime \prime}(z)+c w^{\prime}(z)+f(w(z)) \leq 0 .
$$

In view of (10) this is enough to have

$$
\frac{p(p+1)}{z^{p+2}}-\frac{c p}{z^{p+1}}+\frac{r}{z^{p \beta}} \leq 0
$$


for large $z>>1$, which requires $p+1 \leq p \beta$, that is $\frac{1}{\beta-1} \leq p$. On the other hand we also need the ordering at initial time, which in view of $(9)$, requires $p \leq \alpha$. Hence one needs $\frac{1}{\beta-1} \leq \alpha$, so that the hyperbola separation (8) arises very naturally. Let us now make this formal argument precise.

We define

where $K>1$.

$$
p:=\frac{1}{\beta-1}, \quad w(z):=\frac{K}{z^{p}} \quad \text { for } z \geq z_{0}:=K^{1 / p},
$$

Lemma 3.1. (Supersolutions traveling at constant speed) Let assumptions of Theorem 2.3 hold. Then, for any $K>1$, there is $c>0$ such that

$$
w^{\prime \prime}(z)+c w^{\prime}(z)+f(w(z)) \leq 0, \quad \forall z \geq z_{0} .
$$

Proof. In view of (10), if $z \geq z_{1}:=\left(\frac{K}{s_{0}}\right)^{1 / p}>z_{0}$ then $w(z)=\frac{K}{z^{p}} \leq s_{0}$ so that

$$
w^{\prime \prime}(z)+c w^{\prime}(z)+f(w(z)) \leq \frac{K p(p+1)}{z^{p+2}}-\frac{c K p}{z^{p+1}}+\frac{r K^{\beta}}{z^{p \beta}}=\frac{K p(p+1)}{z^{p+2}}-\frac{c K p-r K^{\beta}}{z^{p+1}} .
$$

Choosing $c>r(\beta-1) K^{\beta-1}$, the above is clearly negative for $z$ large enough, say $z \geq z_{2}$. Last, on the remaining compact region $z_{0} \leq z \leq z_{2}$, we have

$$
\begin{aligned}
w^{\prime \prime}(z)+c w^{\prime}(z)+f(w(z)) & =\frac{K p(p+1)}{z^{p+2}}-\frac{c K p}{z^{p+1}}+f(w(z)) \\
& \leq \frac{K p(p+1)}{z_{0}^{p+2}}-\frac{c K p}{z_{2}^{p+1}}+\|f\|_{L^{\infty}(0,1)} \\
& \leq 0
\end{aligned}
$$

by enlarging $c$ if necessary.

We can now complete the proof of Theorem 2.3. We select $K=\max (1, C)$, where $C>0$ is the constant that appears in (9), and $c>0$ the associated speed given by the above lemma. Then

$$
v(t, x):=\min \left(1, w\left(x-x_{0}+1-c t\right)\right),
$$

is a supersolution of equation (1). Indeed, since 1 solves (1) it is enough to deal with the region where $v(t, x)<1$, that is $z:=x-x_{0}+1-c t>z_{0}$, where it directly follows from the above lemma since $\left(\partial_{t} v-\partial_{x x} v-f(v)\right)(t, x)=\left(-c w^{\prime}-w^{\prime \prime}-f(w)\right)(z)$. Also we have

$$
v(0, x)=\min \left(1, \frac{K}{\left(x-x_{0}+1\right)^{p}}\right) \geq u_{0}(x),
$$

in view of $u_{0} \leq 1$, the assumption on the tail (9), $K \geq C$ and $p=\frac{1}{\beta-1} \leq \alpha$. It follows from the comparison principle that

$$
u(t, x) \leq v(t, x)=\min \left(1, w\left(x-x_{0}+1-c t\right)\right) .
$$

Now, let $\lambda \in(0,1)$ be given. In view of $(7)$, for $t \geq t_{\lambda}$, we can pick $x \in E_{\lambda}(t)$, and the above inequality enforces

$$
x \leq x_{0}-1+\left(\frac{K}{\lambda}\right)^{\beta-1}+c t \leq(c+1) t,
$$

for all $t \geq T_{\lambda}$, if $T_{\lambda} \geq t_{\lambda}$ is sufficiently large. This proves the upper bound in (11). The lower bound in (11) being known since (7), this completes the proof of Theorem 2.3. 


\section{Acceleration despite Allee effect}

In this section, we analyze the situations where the algebraic tail is stronger than the Allee effect, in the sense of (15), so that the solution accelerates. Namely, we prove Theorem 2.5. Notice that, in view of (16) and the comparison principle, we only need to consider the case where

$$
u_{0}(x)=\frac{C}{x^{\alpha}}, \quad \forall x \geq x_{0} .
$$

\subsection{An accelerating small bump as a subsolution}

The main difficulty is to construct a subsolution which has the form of a small bump and travels to the right by accelerating. To do so in a KPP situation, the authors in [19] consider a perturbation of the solution of $\frac{d}{d t} w(t, x)=r w(t, x)$ with $w(0, x)=u_{0}(x)$ as initial data, where $x \in \mathbb{R}$ serves as a parameter. Guided by this approach, we shall rely — in our degenerate situation - on the solution of $\frac{d}{d t} w(t, x)=r w^{\beta}(t, x)$ with $w(0, x)=u_{0}(x)$ as initial data, where $x \in \mathbb{R}$ serves as a parameter. Computations are more involved, and it will turn out that the higher order term of the nonlinearity - typically of the form $f(s)=r s^{\beta}\left(1-s^{\delta}\right)$ - will play a role, so that we first need to assume (24). We start with some preparations.

Let $\varepsilon>0$ small be given. We first make the additional assumption (to be removed in the end of the section)

$$
\beta<1+\delta
$$

We can therefore select a $\rho>0$ such that

$$
\max \left(\frac{\beta r}{1+\delta}, r-\varepsilon\right)<\rho<r
$$

Then define

$$
w(t, x):=\frac{1}{\left(\frac{1}{u_{0}^{\beta-1}(x)}-\rho(\beta-1) t\right)^{\frac{1}{\beta-1}}} \quad \text { for } 0 \leq t<T(x):=\frac{1}{\rho(\beta-1) u_{0}^{\beta-1}(x)},
$$

which solves

$$
\partial_{t} w(t, x)=\rho w^{\beta}(t, x), \quad w(0, x)=u_{0}(x) .
$$

Remark 4.1. Notice that, as $x \rightarrow+\infty$ the interval of existence $(0, T(x))$ of the solution $w(t, x)$ becomes large since, in view of $(23)$,

$$
T(x)=\frac{x^{\alpha(\beta-1)}}{\rho(\beta-1) C^{\beta-1}}, \quad \forall x \geq x_{0} .
$$

Also, since $\alpha(\beta-1)<1$, we will have "enough place" to observe the acceleration phenomenon which, in some sense, is given by $x(t) \sim \mathcal{O}\left(t^{\frac{1}{\alpha(\beta-1)}}\right)$ as $t \rightarrow \infty$, as can be seen in Theorem 2.6.

Straightforward computations yield

$$
\partial_{x x} w(t, x)=g(x) w^{\beta}(t, x)+\beta h(x) w^{2 \beta-1}(t, x),
$$


where

$$
g(x):=\frac{u_{0}^{\prime \prime}(x)}{u_{0}^{\beta}(x)}-\beta \frac{\left(u_{0}^{\prime}(x)\right)^{2}}{u_{0}^{\beta+1}(x)}, \quad h(x):=\frac{\left(u_{0}^{\prime}(x)\right)^{2}}{u_{0}^{2 \beta}(x)} .
$$

In view of $(23)$ and $\beta<1+\frac{1}{\alpha}$, we see that both $g(x)$ and $h(x)$ tend to zero as $x \rightarrow+\infty$. Let us therefore select $x_{1}>x_{0}$ such that

$$
|g(x)|+\beta|h(x)| \leq \frac{r-\rho}{2} \quad \text { and } \quad|g(x)|+(\delta+\beta)|h(x)| \leq \frac{\rho-\frac{r \beta}{1+\delta}}{2}, \quad \forall x \geq x_{1} .
$$

Now, Assumption 2.1 implies that

$$
\kappa:=\inf _{x \in\left(-\infty, x_{1}\right)} u_{0}(x) \in(0,1] .
$$

Last, we select $A>0$ large enough so that

$$
A>\max \left(\frac{1}{\kappa^{\delta}}, \frac{2 r}{1+\delta}\left(\rho-\frac{r \beta}{1+\delta}\right)^{-1}\right),
$$

and

$$
\frac{\delta}{1+\delta} \frac{1}{(A(1+\delta))^{1 / \delta}} \leq s_{0},
$$

where $s_{0}$ is as in (17). Equipped with the above material, we are now in the position to construct the desired subsolution.

Lemma 4.2. (An accelerating subsolution) Let assumptions of Theorem 2.5 hold. Then the function

$$
v(t, x):=\max \left(0, w(t, x)-A w^{1+\delta}(t, x)\right)
$$

is a subsolution of equation $(1)$ in $(0, \infty) \times \mathbb{R}$.

Proof. Since 0 solves (1) it is enough to consider the $(t, x)$ for which $v(t, x)>0$. We therefore need to show

$\mathcal{L} v(t, x):=\partial_{t} v(t, x)-\partial_{x x} v(t, x)-f(v(t, x)) \leq 0 \quad$ when $v(t, x)=w(t, x)-A w^{1+\delta}(t, x)>0$.

This implies in particular that $w(t, x)<1 / A^{1 / \delta}<\kappa$ so that $u_{0}(x)=w(0, x) \leq w(t, x)<\kappa$ since $t \mapsto w(t, x)$ is increasing. In view of the definition of $\kappa$ in (31), this enforces $x \geq x_{1}$. As a result estimates (30) are available. On the other hand $v(t, x) \leq \max _{0 \leq w \leq A^{1 / \delta}} w-A w^{1+\delta}=$ $\frac{\delta}{1+\delta} \frac{1}{(A(1+\delta))^{1 / \delta}} \leq s_{0}$ by (33). Hence, it follows from (17) that

$$
\begin{aligned}
f(v(t, x)) & \geq r v^{\beta}(t, x)\left(1-v^{\delta}(t, x)\right) \\
& \geq r v^{\beta}(t, x)-r w^{\beta+\delta}(t, x) \\
& =r w^{\beta}(t, x)\left(1-A w^{\delta}(t, x)\right)^{\beta}-r w^{\beta+\delta}(t, x) .
\end{aligned}
$$

Then the convexity inequality $\left(1-A w^{\delta}\right)^{\beta} \geq 1-A \beta w^{\delta}$ yields

$$
f(v(t, x)) \geq r w^{\beta}(t, x)-r A \beta w^{\beta+\delta}(t, x)-r w^{\beta+\delta}(t, x) .
$$


Using this, (27), (28), computing $\partial_{t} w^{1+\delta}(t, x)$ and $\partial_{x x} w^{1+\delta}(t, x)$, we arrive at

$$
\begin{aligned}
\mathcal{L} v(t, x) \leq & \rho w^{\beta}(t, x)-g(x) w^{\beta}(t, x)-\beta h(x) w^{2 \beta-1}(t, x)-A \rho(1+\delta) w^{\beta+\delta}(t, x) \\
& +A(1+\delta) g(x) w^{\beta+\delta}(t, x)+A(1+\delta)(\delta+\beta) h(x) w^{2 \beta+\delta-1}(t, x) \\
& -r w^{\beta}(t, x)+r A \beta w^{\beta+\delta}(t, x)+r w^{\beta+\delta}(t, x) .
\end{aligned}
$$

Since $0 \leq w<1$ we have $w^{2 \beta-1} \leq w^{\beta}$ and $w^{2 \beta+\delta-1} \leq w^{\beta+\delta}$, so that

$$
\begin{aligned}
\mathcal{L} v(t, x) \leq & w^{\beta}(t, x)[\rho-r+|g(x)|+\beta|h(x)|] \\
& +w^{\beta+\delta}(x)\left[A(1+\delta)\left(-\rho+\frac{r \beta}{1+\delta}+|g(x)|+(\delta+\beta)|h(x)|\right)+r\right] .
\end{aligned}
$$

The first inequality in (30) implies $\rho-r+|g(x)|+\beta|h(x)| \leq \frac{\rho-r}{2} \leq 0$ and, using the second inequality in (30), we get

$$
\mathcal{L} v(t, x) \leq w^{\beta+\delta}(x)\left[A(1+\delta) \frac{-\rho+\frac{r \beta}{1+\delta}}{2}+r\right] \leq 0,
$$

thanks to (32). Lemma 4.2 is proved.

Since $v(0, x)=\max \left(0, u_{0}(x)-A u_{0}^{1+\delta}(x)\right) \leq u_{0}(x)$, we deduce from the comparison principle that

$$
u(t, x) \geq v(t, x)=\max \left(0, w(t, x)-A w^{1+\delta}(t, x)\right), \quad \forall(t, x) \in[0, \infty) \times \mathbb{R} .
$$

\subsection{Lower bounds on the level sets}

Proof of (18) for small $\lambda$, under assumption (24). Equipped with the above subsolution, whose role is to "lift" the solution $u(t, x)$ on intervals that enlarge with acceleration, we first prove the lower bound (18) on the level sets $E_{\lambda}(t)$ when $\lambda$ is small.

Let us fix

$$
0<\theta<1 / A^{1 / \delta} \text {. }
$$

We claim that, for any $t \geq 0$, there is a unique $y_{\theta}(t) \in \mathbb{R}$ such that $w\left(t, y_{\theta}(t)\right)=\theta$, and moreover $y_{\theta}(t)$ is given by

$$
y_{\theta}(t):=\left(\left(\frac{C}{\theta}\right)^{\beta-1}+\rho C^{\beta-1}(\beta-1) t\right)^{\frac{1}{\alpha(\beta-1)}} .
$$

Indeed, since $\theta<1 / A^{1 / \delta}<\kappa=\inf _{x \in\left(-\infty, x_{1}\right)} u_{0}(x)$ and since $w(t, x) \geq w(0, x)=u_{0}(x)$, for $w(t, y)=\theta$ to hold one needs $y \geq x_{1}$. But, when $y \geq x_{1}>x_{0}$, one can use formula (23) and then solve equation $w(t, y)=\theta$, thanks to expression (26), to find the unique solution (35).

Let us now define the open set

$$
\Omega:=\left\{(t, x), t>0, x<y_{\theta}(t)\right\} .
$$

Let us evaluate $u(t, x)$ on the boundary $\partial \Omega$. For $t>0$, it follows from (34) that

$$
u\left(t, y_{\theta}(t)\right) \geq w\left(t, y_{\theta}(t)\right)-A w^{1+\delta}\left(t, y_{\theta}(t)\right)=\theta-A \theta^{1+\delta}>0 .
$$


On the other hand, for $t=0$ and $x \leq y_{\theta}(0)=\left(\frac{C}{\theta}\right)^{1 / \alpha}$, we have

$$
u(0, x) \geq \inf _{x \leq\left(\frac{C}{\theta}\right)^{1 / \alpha}} u_{0}(x)>0,
$$

in view of Assumption 2.1. As a result $\Theta:=\inf _{(t, x) \in \partial \Omega} u(t, x)>0$. Since $\Theta>0$ is a subsolution for equation (1), it follows from the comparison principle that

$$
u(t, x) \geq \Theta, \quad \forall t \geq 0, \forall x \leq y_{\theta}(t) .
$$

This implies in particular that, for any $0<\lambda<\Theta$, we have, for all $t \geq t_{\lambda}$,

$$
\emptyset \neq E_{\lambda}(t) \subset\left(y_{\theta}(t),+\infty\right) \subset\left(x_{\rho}^{-}(t),+\infty\right), \quad x_{\rho}^{-}(t):=\left(\rho C^{\beta-1}(\beta-1) t\right)^{\frac{1}{\alpha(\beta-1)}},
$$

which implies the lower bound (18) since $\rho>r-\varepsilon$.

Proof of (18) for any $\lambda \in(0,1)$, under assumption (24). Let us now turn to the the case where $\lambda$ is larger than $\Theta$. Let $\Theta \leq \lambda<1$ be given. Let us denote by $v(t, x)$ the solution of (1) with initial data

$$
v_{0}(x):= \begin{cases}\Theta & \text { if } x \leq-1 \\ -\Theta x & \text { if }-1<x<0 \\ 0 & \text { if } x \geq 0 .\end{cases}
$$

It follows from [37, Theorem 1.1] that $\lim _{t \rightarrow \infty} \inf _{x \leq \gamma_{1} t} v(t, x)=1$, for some $\gamma_{1}>0$. In particular there is a time $\tau_{\lambda, \varepsilon}>0$ (this time depends on $\theta$ and therefore on $\varepsilon$ from the above construction of the small bump subsolution) such that

$$
v\left(\tau_{\lambda, \varepsilon}, x\right)>\lambda, \quad \forall x \leq 0 .
$$

On the other hand, it follows from (36) and the definition (38) that

$$
u(T, x) \geq v_{0}\left(x-y_{\theta}(T)\right), \quad \forall T \geq 0, \forall x \in \mathbb{R},
$$

so that the comparison principle yields

$$
u(T+\tau, x) \geq v\left(\tau, x-y_{\theta}(T)\right), \quad \forall T \geq 0, \forall \tau \geq 0, \forall x \in \mathbb{R} .
$$

In view of (39), this implies that

$$
u\left(T+\tau_{\lambda, \varepsilon}, x\right)>\lambda, \quad \forall T \geq 0, \forall x \leq y_{\theta}(T) .
$$

Hence, for any $t \geq T_{\lambda, \varepsilon}^{1}:=\max \left(\tau_{\lambda, \varepsilon}, t_{\lambda}\right)$, if we pick a $x \in E_{\lambda}(t)$ then the above implies $x>y_{\theta}\left(t-\tau_{\lambda, \varepsilon}\right)$, that is

$$
x>\left(\left(\frac{C}{\theta}\right)^{\beta-1}-\rho C^{\beta-1}(\beta-1) \tau_{\lambda, \varepsilon}+\rho C^{\beta-1}(\beta-1) t\right)^{\frac{1}{\alpha(\beta-1)}} \geq\left((r-\varepsilon) C^{\beta-1}(\beta-1) t\right)^{\frac{1}{\alpha(\beta-1)}},
$$

for all $t \geq T_{\lambda, \varepsilon}$, with $T_{\lambda, \varepsilon}>0$ sufficiently large (recall that $\rho>r-\varepsilon$ ). This proves the lower bound (18) when $\Theta \leq \lambda<1$ and concludes the proof of Theorem 2.5, under the additional assumption (24).

Relaxing the additional assumption (24). When $\beta<1+\delta$ does not hold, let us pick $\delta^{*}>0$ such that $\beta<1+\delta^{*}$ and define $r^{*}:=r-\frac{\varepsilon}{2}$. It follows from (17) that there is $s_{0}^{*} \in(0,1)$ such that

$$
f(s) \geq r^{*} s^{\beta}\left(1-s^{\delta^{*}}\right), \quad \forall 0 \leq s \leq s_{0}^{*} .
$$

Hence, from the above analysis, (18) is available with $r^{*}$ in place of $r$. This concludes the proof of Theorem 2.5. 


\section{Upper bounds on the level sets when acceleration}

In this section, we sandwich the level sets $E_{\lambda}(t)$ of the solution $u(t, x)$ when acceleration occurs, namely we prove Theorem 2.6. In view of Theorem 2.5, it only remains to prove the upper estimate in (22).

Let $\lambda \in(0,1)$ and $\varepsilon>0$ small be given. Up to enlarging $x_{0}>1$ which appears in $(20)$, we can assume without loss of generality that

$$
\frac{\alpha(\alpha+1+2 \beta \alpha)}{x_{0}^{2}} \leq \frac{\varepsilon}{2}
$$

and $\frac{\bar{C}}{x_{0}^{\alpha}}<1$. Then, up to enlarging $\bar{C}>0$ which also appears in (20), we can assume without loss of generality that

$$
\frac{\bar{C}}{x_{0}^{\alpha}}=1 .
$$

Now, for these $x_{0}>1$ and $\bar{C}>0$, in view of (20) and the comparison principle, it is enough to prove the upper bound in (22) when

$$
u_{0}(x)=\frac{\bar{C}}{x^{\alpha}}, \quad \forall x \geq x_{0} .
$$

Let us select

$$
\rho:=\bar{r}+\frac{\varepsilon}{2}
$$

We then define

$$
\psi(t, x):=\min \left(1, w(t, x):=\frac{1}{\left(\frac{1}{u_{0}^{\beta-1}(x)}-\rho(\beta-1) t\right)^{\frac{1}{\beta-1}}}\right),
$$

where $w(t, x)$ is as in (26). We claim that $\psi$ is a supersolution for equation (1) in the domain $\Omega:=(0, \infty) \times\left(x_{0},+\infty\right)$. Indeed, since 1 solves $(1)$, it suffices to consider the points $(t, x)$ where $\psi(t, x)=w(t, x)<1$. In view of

$$
\partial_{t} w(t, x)=\rho w^{\beta}(t, x)=\left(\bar{r}+\frac{\varepsilon}{2}\right) w^{\beta}(t, x),
$$

and inequality (21), some straightforward computations yield

$$
\begin{aligned}
\partial_{t} w(t, x) & -\partial_{x x} w(t, x)-f(w(t, x)) \\
& \geq \frac{\varepsilon}{2} w^{\beta}(t, x)-g(x) w^{\beta}(t, x)-\beta h(x) w^{2 \beta-1}(t, x) \\
& \geq w^{\beta}(t, x)\left(\frac{\varepsilon}{2}-|g(x)|-\beta|h(x)|\right),
\end{aligned}
$$

since $0<w(t, x)<1$, and where $g(x)$ and $h(x)$ were defined in (29), that is

$$
g(x):=\frac{u_{0}^{\prime \prime}(x)}{u_{0}^{\beta}(x)}-\beta \frac{\left(u_{0}^{\prime}(x)\right)^{2}}{u_{0}^{\beta+1}(x)}, \quad h(x):=\frac{\left(u_{0}^{\prime}(x)\right)^{2}}{u_{0}^{2 \beta}(x)} .
$$


In view of expression (42), some straightforward computations yield that, for any $x \geq x_{0}$,

$$
\begin{aligned}
|g(x)|+\beta & h(x) \mid \\
\leq & \frac{\alpha(\alpha+1)}{\bar{C}^{\beta-1} x^{\alpha+2-\alpha \beta}}+\beta\left(\frac{\alpha^{2}}{\bar{C}^{\beta-1} x^{\alpha+2-\alpha \beta}}+\frac{\alpha^{2}}{\bar{C}^{2 \beta-2} x^{2 \alpha+2-2 \alpha \beta}}\right) \\
\leq & \frac{\alpha(\alpha+1)}{\bar{C}^{\beta-1} x_{0}^{\alpha+2-\alpha \beta}}+\beta\left(\frac{\alpha^{2}}{\bar{C}^{\beta-1} x_{0}^{\alpha+2-\alpha \beta}}+\frac{\alpha^{2}}{\bar{C}^{2 \beta-2} x_{0}^{2 \alpha+2-2 \alpha \beta}}\right)
\end{aligned}
$$

since both $\alpha+2-\alpha \beta$ and $2 \alpha+2-2 \alpha \beta$ are positive thanks to $\beta<1+\frac{1}{\alpha}$. Now in view of (41), we get

$$
|g(x)|+\beta|h(x)| \leq \frac{\alpha(\alpha+1)}{x_{0}^{2}}+\beta\left(\frac{\alpha^{2}}{x_{0}^{2}}+\frac{\alpha^{2}}{x_{0}^{2}}\right) \leq \frac{\varepsilon}{2},
$$

in virtue of (40). It therefore follows from (43) that, for any $(t, x) \in \Omega$ such that $w(t, x)<1$,

$$
\partial_{t} w(t, x)-\partial_{x x} w(t, x)-f(w(t, x)) \geq 0,
$$

which proves our claim that $\psi$ is a supersolution of (1) in $\Omega$.

Let us now have a look at the boundary $\partial \Omega=\{0\} \times\left[x_{0},+\infty\right) \cup(0, \infty) \times\left\{x_{0}\right\}$. For $t=0, x \geq x_{0}$, we have $w(0, x)=u_{0}(x)=u(0, x)$, whereas for $t>0, x=x_{0}$ we have $w\left(t, x_{0}\right)=\frac{1}{(1-\rho(\beta-1) t)^{\frac{1}{\beta-1}}} \geq 1 \geq u\left(t, x_{0}\right)$. Hence $\psi(t, x) \geq u(t, x)$ for any $(t, x) \in \partial \Omega$. We deduce from the comparison principle that

$$
u(t, x) \leq \psi(t, x) \leq w(t, x), \quad \forall(t, x) \in[0, \infty) \times\left[x_{0},+\infty\right) .
$$

Now, we can define $T_{\lambda, \frac{r}{2}} \geq t_{\lambda}$ as in the conclusion (18) of Theorem 2.5. For $t \geq T_{\lambda, \frac{r}{2}}$, let us pick a $x \in E_{\lambda}(t)$. We know from (18) that $x \geq\left(\frac{r}{2} C^{\beta-1}(\beta-1) t\right)^{\frac{1}{\alpha(\beta-1)}} \rightarrow+\infty$ as $t \rightarrow \infty$ so, up to enlarging $T_{\lambda, \frac{r}{2}}$, we can assume that $x \geq x_{0}$. It therefore follows from (44) that $w(t, x) \geq \lambda$ which, using the expression for $w(t, x)$ transfers into

$$
\begin{aligned}
x & \leq\left(\left(\frac{\bar{C}}{\lambda}\right)^{\beta-1}+\left(\bar{r}+\frac{\varepsilon}{2}\right) \bar{C}^{\beta-1}(\beta-1) t\right)^{\frac{1}{\alpha(\beta-1)}} \\
& <\left((\bar{r}+\varepsilon) \bar{C}^{\beta-1}(\beta-1) t\right)^{\frac{1}{\alpha(\beta-1)}}=: x^{+}(t),
\end{aligned}
$$

for $t \geq \bar{T}_{\lambda, \varepsilon}$, with $\bar{T}_{\lambda, \varepsilon} \geq T_{\lambda, \frac{r}{2}}$ chosen sufficiently large. This proves the upper bound in (22) and therefore concludes the proof of Theorem 2.6.

Acknowledgement. The author thanks J. Coville (for suggesting the issue under consideration), A. Ducrot (for pointing reference [37]), G. Faye and J.-M. Roquejoffre (for pointing reference [15]), and Q. Griette (for making Figure 1).

\section{References}

[1] F. Achleitner And C. Kuehn, Traveling waves for a bistable equation with nonlocaldiffusion, preprint. 
[2] M. Alfaro And J. Coville, Propagation phenomena in nonlocal monostable equations: traveling waves vs. acceleration, in preparation.

[3] D. G. Aronson and H. F. Weinberger, Multidimensional nonlinear diffusion arising in population genetics, Adv. in Math., 30 (1978), pp. 33-76.

[4] P. W. Bates, P. C. Fife, X. Ren, And X. Wang, Traveling waves in a convolution model for phase transitions, Arch. Rational Mech. Anal., 138 (1997), pp. 105-136.

[5] J. W. Bebernes, C. LI, AND Y. LI, Travelling fronts in cylinders and their stability, Rocky Mountain J. Math., 27 (1997), pp. 123-150.

[6] H. Berestycki, F. Hamel, And G. Nadin, Asymptotic spreading in heterogeneous diffusive excitable media, J. Funct. Anal., 255 (2008), pp. 2146-2189.

[7] H. Berestycki, F. Hamel, And N. Nadirashvili, The speed of propagation for KPP type problems. I. Periodic framework, J. Eur. Math. Soc. (JEMS), 7 (2005), pp. 173-213.

[8] — The speed of propagation for KPP type problems. II. General domains, J. Amer. Math. Soc., 23 (2010), pp. 1-34.

[9] X. Cabré and J.-M. Roquejoffre, The influence of fractional diffusion in FisherKPP equations, Comm. Math. Phys., 320 (2013), pp. 679-722.

[10] X. Chen, Existence, uniqueness, and asymptotic stability of traveling waves in nonlocal evolution equations, Adv. Differential Equations, 2 (1997), pp. 125-160.

[11] J. CoviLLE, Travelling fronts in asymmetric nonlocal reaction diffusion equation: the bistable and ignition case, preprint.

[12] J. Coville And L. Dupaigne, On a non-local equation arising in population dynamics, Proc. Roy. Soc. Edinburgh Sect. A, 137 (2007), pp. 727-755.

[13] R. A. Fisher, The wave of advance of advantageous genes, Ann. of Eugenics, 7 (1937), pp. 355-369.

[14] J. GARNIER, Accelerating solutions in integro-differential equations, SIAM J. Math. Anal., 43 (2011), pp. 1955-1974.

[15] C. Gui And T. Huan, Traveling wave solutions to some reaction diffusion equations with fractional laplacians, to appear in Calc. Var.

[16] C. Gui And M. ZhaO, Traveling wave solutions of Allen-Cahn equation with a fractional laplacian, to appear in Ann. I. H. Poincaré.

[17] K. P. Hadeler And F. Rothe, Travelling fronts in nonlinear diffusion equations, J. Math. Biol., 2 (1975), pp. 251-263.

[18] F. Hamel and G. Nadin, Spreading properties and complex dynamics for monostable reaction-diffusion equations, Comm. Partial Differential Equations, 37 (2012), pp. 511537. 
[19] F. Hamel And L. Roques, Fast propagation for KPP equations with slowly decaying initial conditions, J. Differential Equations, 249 (2010), pp. 1726-1745.

[20] F. Hamel And Y. SiRe, Spreading speeds for some reaction-diffusion equations with general initial conditions, SIAM J. Math. Anal., 42 (2010), pp. 2872-2911.

[21] Y. Kametaka, On the nonlinear diffusion equation of Kolmogorov-Petrovskii-Piskunov type, Osaka J. Math., 13 (1976), pp. 11-66.

[22] J. I. KANEL', Stabilization of the solutions of the equations of combustion theory with finite initial functions, Mat. Sb. (N.S.), 65 (107) (1964), pp. 398-413.

[23] A. L. Kay, J. A. Sherratt, and J. B. McLeod, Comparison theorems and variable speed waves for a scalar reaction-diffusion equation, Proc. Roy. Soc. Edinburgh Sect. A, 131 (2001), pp. 1133-1161.

[24] A. Kiselev And L. RYzhik, Enhancement of the traveling front speeds in reactiondiffusion equations with advection, Ann. Inst. H. Poincaré Anal. Non Linéaire, 18 (2001), pp. 309-358.

[25] A. N. Kolmogorov, I. G. Petrovsky, and N. S. Piskunov, Etude de l'équation de la diffusion avec croissance de la quantité de matière et son application à un problème biologique, Bull. Univ. Etat Moscou, Sér. Inter. A 1 (1937), pp. 1-26.

[26] J. A. Leach, D. J. Needham, And A. L. Kay, The evolution of reaction-diffusion waves in a class of scalar reaction-diffusion equations: algebraic decay rates, Phys. D, 167 (2002), pp. 153-182.

[27] H. P. McKean, Application of Brownian motion to the equation of KolmogorovPetrovskii-Piskunov, Comm. Pure Appl. Math., 28 (1975), pp. 323-331.

[28] D. J. Needham And A. N. Barnes, Reaction-diffusion and phase waves occurring in a class of scalar reaction-diffusion equations, Nonlinearity, 12 (1999), pp. 41-58.

[29] J.-M. Roquejoffre, Eventual monotonicity and convergence to travelling fronts for the solutions of parabolic equations in cylinders, Ann. Inst. H. Poincaré Anal. Non Linéaire, 14 (1997), pp. 499-552.

[30] J. A. Sherratt And B. P. Marchant, Algebraic decay and variable speeds in wavefront solutions of a scalar reaction-diffusion equation, IMA J. Appl. Math., 56 (1996), pp. 289-302.

[31] K. UChIYAma, The behavior of solutions of some nonlinear diffusion equations for large time, J. Math. Kyoto Univ., 18 (1978), pp. 453-508.

[32] H. F. WeInBERGer, Long-time behavior of a class of biological models, SIAM J. Math. Anal., 13 (1982), pp. 353-396.

[33] J. XIN, Existence and nonexistence of traveling waves and reaction-diffusion front propagation in periodic media, J. Statist. Phys., 73 (1993), pp. 893-926.

[34] _ _ Front propagation in heterogeneous media, SIAM Rev., 42 (2000), pp. 161-230. 
[35] G.-B. Zhang, Global stability of wavefronts with minimal speeds for nonlocal dispersal equations with degenerate nonlinearity, Nonlinear Anal., 74 (2011), pp. 6518-6529.

[36] G.-B. Zhang, W.-T. LI, AND Z.-C. WANG, Spreading speeds and traveling waves for nonlocal dispersal equations with degenerate monostable nonlinearity, J. Differential Equations, 252 (2012), pp. 5096-5124.

[37] A. Zlatoš, Quenching and propagation of combustion without ignition temperature cutoff, Nonlinearity, 18 (2005), pp. 1463-1475. 